University of Nebraska - Lincoln

DigitalCommons@University of Nebraska - Lincoln

\title{
Creation of a high-amylose durum wheat through mutagenesis of starch synthase II (SSIIa)
}

\author{
A.C. Hogg \\ Montana State University \\ K. Gause \\ Montana State University \\ P. Hofer \\ Montana State University \\ J.M. Martin \\ Montana State University \\ Robert A. Graybosch \\ University of Nebraska-Lincoln, bob.graybosch@ars.usda.gov \\ See next page for additional authors
}

Follow this and additional works at: https://digitalcommons.unl.edu/usdaarsfacpub

Hogg, A.C.; Gause, K.; Hofer, P.; Martin, J.M.; Graybosch, Robert A.; Hansen, L.E.; and Giroux, M.J., "Creation of a high-amylose durum wheat through mutagenesis of starch synthase II (SSIla)" (2013). Publications from USDA-ARS / UNL Faculty. 1152.

https://digitalcommons.unl.edu/usdaarsfacpub/1152

This Article is brought to you for free and open access by the U.S. Department of Agriculture: Agricultural Research Service, Lincoln, Nebraska at DigitalCommons@University of Nebraska - Lincoln. It has been accepted for inclusion in Publications from USDA-ARS / UNL Faculty by an authorized administrator of DigitalCommons@University of Nebraska - Lincoln. 
Authors

A.C. Hogg, K. Gause, P. Hofer, J.M. Martin, Robert A. Graybosch, L.E. Hansen, and M.J. Giroux 


\title{
Creation of a high-amylose durum wheat through mutagenesis of starch synthase II (SSIIa)
}

\author{
A.C. Hogg ${ }^{a}$, K. Gause ${ }^{\mathrm{a}, 1}$, P. Hofer ${ }^{\mathrm{a}}$, J.M. Martin ${ }^{\mathrm{a}}$, R.A. Graybosch ${ }^{\mathrm{b}}$, L.E. Hansen ${ }^{\mathrm{b}}$, M.J. Giroux ${ }^{\mathrm{a}, *}$ \\ ${ }^{a}$ Department of Plant Sciences and Plant Pathology, 119 Plant Bioscience Building, Montana State University, Bozeman, MT 59717-3150, USA \\ ${ }^{\mathrm{b}}$ USDA-ARS, 137 Keim Hall, East Campus, University of Nebraska, Lincoln, NE 68583-0937, USA
}

\section{A R T I C L E I N F O}

\section{Article history:}

Received 24 October 2012

Received in revised form

31 December 2012

Accepted 7 January 2013

\section{Keywords:}

Starch

Amylose

Wheat

RNA-seq

\begin{abstract}
A B S T R A C T
In cereal seeds, mutations in one or more starch synthases lead to decreased amylopectin and increased amylose content. Here, the impact of starch synthase IIa (SSIIa or SGP-1) mutations upon durum starch was investigated. A screen of durum accessions identified two lines lacking SGP-A1, the A genome copy of SGP-1. The two lines were determined to carry a 29 bp deletion in the first exon of SSIIa. The SGP-A1 nulls were crossed with the durum variety 'Mountrail' and $F_{5}$ derived SGP-A1 null progeny lines were treated with EMS. From each EMS population, one SGP-B1 null mutation was recovered with each being a missense mutation. Each of the SGP-1 nulls was found to have large increases in amylose content and reduced binding of SGP-2 and SGP-3 to the interior of starch granules. RNA-Seq was used to examine the impact the loss of SGP-1 has upon other starch biosynthetic genes. Significant increases in transcript levels of several starch biosynthetic genes were observed in SGP-1 nulls relative to Mountrail. The resultant high amylose durums may prove useful in the creation of value added pasta with increased firmness and reduced glycemic index.
\end{abstract}

(c) 2013 Elsevier Ltd. All rights reserved.

\section{Introduction}

In bread wheat (Triticum aestivum L.), amylose accounts for approximately $25 \%$ of starch with amylopectin accounting for the other 75\% (reviewed in Tetlow, 2006). The "waxy" proteins (granule bound starch synthase I) encoded by the $W x$ genes are solely responsible for amylose synthesis after the production of ADPglucose by ADP-glucose pyrophosphorylase (AGPase) (reviewed in Tetlow, 2006). In contrast, amylopectin synthesis involves a host of enzymes such as starch synthases (SS) I, II, III, IV, starch branching enzymes (SBE) I and II, and starch de-branching enzymes (reviewed in Tetlow, 2006).

Several starch biosynthetic proteins remain bound to the interior of starch granules with a subset of these proteins designated the starch granule proteins (SGPs). Using SDS-PAGE, Yamamori and Endo (1996) separated the SGPs from bread wheat starch into SGP1 , SGP-2, SGP-3 and WX. The SGP-1 fraction was further resolved into SGP-A1, SGP-B1, and SGP-D1 and the associated genes localized

\footnotetext{
Abbreviations: FSP, flour swelling power; SGP, starch granule protein.

* Corresponding author. Tel.: +1 406994 7877; fax: +1 4069947600.

E-mail address: mgiroux@montana.edu (M.J. Giroux).

1 Current address: Department of Plant, Soil, and Environmental Sciences, University of Maine, Orono, Maine 04469-5722, USA.
}

to the homoeologous group 7 chromosomes (Yamamori and Endo, 1996). SGP-1 proteins are isoforms of SSII encoded by the genes SSIIa-A, SSIIa-B, SSIIa-D on the short arms of group 7 chromosomes (Li et al., 1999).

A survey of hexaploid wheat germplasm identified lines lacking SGP-A1, SGP-B1, or SGP-D1 (Yamamori and Endo, 1996) which were crossed to create an SGP-1 null (Yamamori et al., 2000). The SGP-1 null had a $29 \%$ increase in amylose content (37.3\% null vs. $28.9 \%$ wild-type), deformed starch granules, reduced starch content, and reduced binding of SGP-2 and SGP-3 to starch granules. These SGP1 mutations were later shown to reduce starch binding without impacting SGP protein expression levels (Kosar-Hashemi et al., 2007). Lafiandra et al. (2010) reported that SGP-1 null lines created from crosses between the durum (Triticum turgidum ssp. durum) cultivar 'Svevo' and hexaploid SGP-A1/B1 null lines (Yamamori and Endo, 1996) had an 89\% increase in amylose content compared to Svevo (43.6\% SGP-1 null vs $23 \%$ wild-type) as well as reduced binding of SGP-2 and SGP-3. Elimination of SbeIIa in durum through RNA interference also resulted in increased amylose ranging from $+29 \%$ to $+200 \%$ (24\% wild-type vs. $31-75 \%$ SbeIIa RNAi lines) (Sestili et al., 2010b). The very high amylose results observed by Sestili et al. (2010b) may not be due solely to SbeIIa expression reduction since Sbella mutants have amylose level increases similar to those of SSIIa mutations (28\% sbeIIa versus $23 \%$ wild-type) (Hazard et al., 2012). 
To date a detailed expression profile of starch synthesis genes in an SGP-1 null background has not been reported. RNA-Seq is an emerging method that allows for gene expression analysis at the transcript level and, compared to other methods, has a greater sequencing sensitivity, a large dynamic range, and the ability to distinguish between differing alleles or isoforms of an expressed gene (Marioni et al., 2008). RNA-Seq is therefore an ideal method to determine the effect a null SGP-1 genotype has on expression of other starch synthesis genes.

Cereals with high amylose content are desirable because they have more resistant starch. Resistant starch is starch that resists break down slowly and thus acts more like dietary fiber while promoting microbial fermentation (reviewed in Nugent, 2005). The SGP1 null alleles (Yamamori and Endo, 1996) were introgressed into the Italian bread cultivar 'N11' and whole grain was prepared into bread which was found to have increased resistant starch and decreased glycemic index relative to standard whole wheat breads (Hallstrom et al., 2011). Similarly, products prepared from ssIla barley have been shown to have a reduced glycemic index and improve bowel health indices (Bird et al., 2008; King et al., 2008). Rats fed SbelIa RNAi seeds with an amylose content of $\sim 80 \%$ also showed significant improvements in bowel health indices (Regina et al., 2006).

In addition to the positive impact of increased amylose upon glycemic index, higher amylose might enhance durum product quality. Pasta that is firmer when cooked is often preferred as it resists overcooking and high amylose should increase pasta firmness. Our goal here was to develop a high-amylose durum line through the creation of SSIIa mutations and to examine the effect an SGP-1 null genotype has on the expression of other starch biosynthetic genes.

\section{Materials and methods}

\subsection{Creation and screening of a mutagenized durum wheat population}

Durum wheat accessions obtained from the USDA National Small Grains Collection (NSGC, Aberdeen, ID) and the International Center for Agricultural Research in the Dry Areas (ICARDA) were screened for SGP-1 mutations using SDS-PAGE of SGPs (see below). From the 200 NSGC Triticum durum core collection accessions screened, one line, PI 330546, lacked SGP-A1 and none lacked SGPB1. From the 55 ICARDA T. durum accessions screened, one line, IG 86304, lacked SGP-A1 and none lacked SGP-B1. These two lines were crossed independently with the cultivar "Mountrail" (PVP 9900266) (Elias and Miller, 2000) and progeny lines advanced via single seed descent to the $F_{5}$ generation. Seed from lines homozygous for the SGP-A1 null trait that had seed and plant characteristics similar to Mountrail from each cross were then treated with ethyl methanesulfonate (EMS) as described in Feiz et al. (2009) with the exception that 0.5\% EMS was used and plants were advanced two generations in the greenhouse to the $M_{1}: M_{2}$ generation. Seed from 294 Mountrail/PI $330546 \mathrm{M}_{1}$ lines and 196 Mountrail/IG $86304 \mathrm{M}_{1}$ lines were pre-screened for potential SSIIa$B$ mutations using the flour swelling power (FSP) test reported by Martin et al. (2008) who found that increased amylose content is associated with decreased FSP.

\subsection{Starch extraction}

For each selected low flour swelling power (FSP) genotype and parental controls, four seeds were ground in a Braun coffee mill (Proctor Gamble, Cincinnati, $\mathrm{OH}$ ) for $10 \mathrm{~s}$ and then placed in a $2 \mathrm{ml}$ microcentrifuge tube along with two $6.5 \mathrm{~mm}$ zirconia balls and agitated for $30 \mathrm{~s}$ in a Mini-beadbeater-96. The zirconia balls were removed from the microcentrifuge tubes and $1.0 \mathrm{ml}$ of $0.1 \mathrm{M} \mathrm{NaCl}$ was added to the whole grain flour and samples were incubated for $30 \mathrm{~min}$ at room temperature. After $30 \mathrm{~min}$, a dough ball was made by mixing the wet flour using a plastic Kontes Pellet Pestle (Kimble Chase, Vineland, $\mathrm{NJ}$ ) and the gluten ball was removed from the samples after pressing out the starch. The liquid starch suspension was then transferred to a new pre-weighed $2.0 \mathrm{ml}$ tube and $0.5 \mathrm{ml} \mathrm{ddH_{2 }} \mathrm{O}$ was added to the remnant starch pellet in the first tube. The first tube was vortexed, left to settle for $1 \mathrm{~min}$ and the liquid starch suspension transferred to the second tube. The starch suspension containing tubes were centrifuged at $5000 \mathrm{~g}$ and the liquid was aspirated off. Next, $0.5 \mathrm{ml}$ of SDS extraction buffer ( $55 \mathrm{mM}$ Tris$\mathrm{Cl}$ pH 6.8, 2.3\% SDS, 5\% BME, 10\% glycerol) was added, the samples were vortexed till suspended, and then centrifuged at $5000 \mathrm{~g}$. The SDS buffer was aspirated off and the SDS buffer extraction was repeated once more. Then, $0.5 \mathrm{ml}$ of $80 \%(\mathrm{w} / \mathrm{v}) \mathrm{CsCl}$ was added to the starch pellets, samples were vortexed till suspended, and centrifuged at $7500 \mathrm{~g}$. The $\mathrm{CsCl}$ solution was aspirated off and the starch

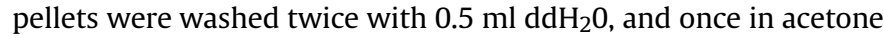
with centrifugation speeds of $10,000 \mathrm{~g}$. After supernatant aspiration, the starch pellets were left to dry overnight in a fume hood.

\subsection{SDS-PAGE of starch granule proteins}

To purified starch, $7.5 \mu$ of SDS loading buffer (SDS extraction buffer plus bromophenol blue) was added per mg of starch. Samples were heated for $15 \mathrm{~min}$ at $70{ }^{\circ} \mathrm{C}$, vortexed, centrifuged for $1 \mathrm{~min}$ at $10,000 \mathrm{~g}$, and then $40 \mu \mathrm{l}$ of sample was loaded in a $203 \times 203 \times 0.75 \mathrm{~mm} \mathrm{10 \% (w/v)} \mathrm{acrylamide} \mathrm{gel} \mathrm{prepared} \mathrm{using}$ a $30 \%$ acrylamide $/ 0.135 \%$ piperazine diacrylamide (PDA) w/v stock solution. The gel had a $4 \% \mathrm{w} / \mathrm{v}$ acrylamide stacking gel prepared using a $30 \%$ acrylamide/0.8\% PDA w/v stock solution. Gels were run at $25 \mathrm{~mA} /$ gel for $45 \mathrm{~min}$ and then $35 \mathrm{~mA} /$ gel for $3 \mathrm{~h}$ and proteins were visualized by silver staining.

\subsection{PCR screening for mutations in SSIIa-A and SSIIa-B}

Leaf tissue from $\mathrm{M}_{2}$ plants suspected of having ssIIa- $B$ mutations and parental lines was collected from two-three leaf stage seedlings and stored at $-80^{\circ} \mathrm{C}$. DNA was isolated from seedlings as previously described (Feiz et al., 2009). Coding regions of SSIIa-A and SSIIa-B were amplified and sequenced from duplicate DNA samples using previously described primers and PCR conditions (Shimbata et al., 2005; Sestili et al., 2010a). Amplicons were analyzed for single nucleotide polymorphisms using Seqman Pro in the Lasergene 10.1 Suite (DNASTAR, Madison, WI). The two durum high amylose (DHA) SGP-1 double mutants discovered were DHA175, from the Mountrail/PI 330546 cross and DHA55, from the Mountrail/IG 86304 cross.

\subsection{Differential scanning calorimetry}

For random SGP-A1 wild type and SGP-A1 null $\mathrm{F}_{5}$ derived lines from Mountrail/PI 330546, DHA175, and DHA55 differential scanning calorimeter (DSC) analysis was carried out using a Pyris 7 Diamond DSC (Perkin Elmer, Norwalk CT, USA) as previously described (Hansen et al., 2010). Three biological replicates were run in triplicate for each genotype. Amylose was determined via DSC using the method described in Polaske et al. (2005).

\subsection{Colorimetric amylose determination and total starch determination}

Purified starch from three biological replicates of SGP-A1 wild type and SGP-A1 null $F_{5}$ derived lines from Mountrail/PI 330546, DHA175, and DHA55 was used for amylose analysis. Amylose was 
determined using a colorimetric assay whereby $2 \mathrm{mg}$ of purified starch was suspended in a $1 \mathrm{~mL}$ iodine solution (90\% Dimethyl sulfoxide; $1.52 \mathrm{~g} / \mathrm{L}$ iodine) in a $2 \mathrm{~mL}$ microcentrifuge tube which was mixed at $1200 \mathrm{rpm}$ at $95{ }^{\circ} \mathrm{C}$ for $30 \mathrm{~min}$ in a Thermomixer (Eppendorf, Hamburg, Germany). Samples were allowed to cool and then $20 \mu \mathrm{L}$ of sample was removed and placed in a 96-well plate with $180 \mu \mathrm{L}$ of $\mathrm{ddH}_{2} \mathrm{O}$. Samples were agitated on a plate mixer at $900 \mathrm{rpm}$ for $2 \mathrm{~min}$ and then immediately read at $620 \mathrm{~nm}$ on a SPECTRAmax Plus 384 (Molecular Devices, Sunnyvale, CA). A standard curve created using purified potato amylose (Sigma, St. Louis, MO, USA) was used to estimate amylose content. Total starch was determined on whole grain flours from three biological reps of SGP-A1 wild type and SGP-A1 null $F_{5}$ derived lines from Mountrail/ PI 330546, DHA175, and DHA55 following previously described methods (Smith and Zeeman, 2006).

\subsection{Microscopic analysis of starch granules}

Purified starch from SGP-A1 wild type and SGP-A1 null $F_{5}$ derived lines from Mountrail/PI 330546, DHA175, and DHA55 were obtained from three biological replicates per sample using the methods described above. Individual starch samples were placed on carbon tape which was then sputtered with iridium $(20 \mathrm{~mA}$ for $30 \mathrm{~s}$ ) and photographed using a Zeiss Supra 55 VP field emission gun-SEM (Carl Zeiss Microscopy, Peabody, MA).

\subsection{Starch synthesis gene expression analysis via RNA-Seq}

To analyze expression levels of starch synthesis genes, developing seeds 14 days post anthesis were collected from Mountrail, DHA55, and DHA175 and stored at $-80{ }^{\circ} \mathrm{C}$. For each genotype, developing seeds were collected from three separate plants, with each plant sample composed of four seeds from the middle of three different spikes (12 seeds total). Total RNA was extracted from the immature kernels after pre-grinding in liquid $\mathrm{N}_{2}$ using an RNeasy Plant Mini Kit (Qiagen, Valencia, CA) after first pre-extracting each sample to remove excess starch. To accomplish this, one hundred $\mathrm{mg}$ of seed powder was transferred to a pre-chilled $1.5 \mathrm{~mL}$ tube and $0.5 \mathrm{~mL}$ of RNA extraction buffer (100 mM Tris pH 8.0, $150 \mathrm{mM} \mathrm{LiCl}$, $50 \mathrm{mM}$ EDTA, 1.5\% (w/v) SDS, 0.15\% (v/v) BME) was added and samples were vortexed until homogenous. Next, $0.25 \mathrm{~mL}$ of $1: 1(\mathrm{v} / \mathrm{v})$ phenol-chloroform ( $\mathrm{pH} 4.7$ ) was added and samples were mixed by inversion followed by centrifugation at $13,000 \mathrm{~g}$ for $15 \mathrm{~min}$ at room temperature. The supernatant was transferred to a QIAshredder spin column and total RNA was extracted per the manufacturer's instructions. Total RNA was quantified and its quality assessed using a Bioanalyzer (Agilent Technologies, Santa Clara, CA). For RNA-Seq analysis, one $\mu \mathrm{g}$ of total RNA was used for the creation of cDNA libraries using TruSeq RNA-Seq library kits (Illumina, San Diego, CA). Amplicons from cDNA libraries were sequenced as single $50 \mathrm{bp}$ reads using a LifeTech SOLiD 5500xl (Life Technologies, Carlsbad, CA). RNA-Seq data was analyzed using Q-Seq in ArrayStar v5.0 (DNASTAR, Madison, WI). Genes of interest were selected from the NCBI database for analysis with the match settings in QSeq set to $100 \%$ for at least $40 \mathrm{bp}$ with mer minimization turned off. All other settings were left to default and sequences were normalized using the Reads Per Kilobase of exon model per Million mapped reads (RPKM) method. Resultant linear counts were then further normalized to the expression levels of the house keeping gene glyceraldehyde-3-phosphate dehydrogenase (Ga3pd). Student's t-tests were used to compare expression levels between Mountrail and the two ssIIa null genotypes, DHA55 and DHA175.

\section{Results}

\subsection{Screening of EMS mutagenized durum lines}

$M_{1}$ seed from EMS treated SGP-A1 nulls from the crosses of Mountrail/PI 330546 and Mountrail/IG 86304 was screened indirectly for mutations in SSIIa-B using an FSP test (Table 1). Lines that had an FSP of less than $6.5\left(\mathrm{~g} \mathrm{~g}^{-1}\right)$ were selected for analysis of SGPs via SDS-PAGE. One line from the Mountrail/PI 330546 cross, DHA175 was lacking SGP-A1/B1, SGP-2 and SGP-3 and line DHA55 from the Mountrail/IG 86304 cross had an SGP-B1 band that was approximately half the intensity of the Mountrail/IG 86304 wildtype control (data not shown), indicating a potential heterozygote. DHA55 was confirmed to be an SGP-1 wild type/null heterozygote using SDS-PAGE of the SGPs from $\mathrm{M}_{2: 3}$ plants. Starch granule proteins from a wild type and SGP-A1 null line derived from Mountrail/ PI 330546, DHA175, and a homozygous SGP-1 double null DHA55 were then analyzed via SDS-PAGE using a dilution series to examine the effect of the SGP-1 nulls on the binding of other SGPs (Fig. 1). In both DHA175 and DHA55 the SGP-A1 and SGP-B1 bands were absent and the SGP-2 and SGP-3 bands were reduced to less than $10 \%$ of the wild-type control. Wx band intensity appeared normal in both SGP-1 double nulls. In the SGP-A1 null control, none of the other SGP bands appeared altered. FSP of the SGP-1 double nulls was significantly lower than their SGP-A1 null sister lines (Table 1).

\subsection{Seed characteristics}

The SGP-A1 null genotype had reduced individual seed weight relative to the SGP-A1 wild type control group with even greater reductions in seed weight in the SGP-1double null (Table 1 ). The SGP-1 double null also had reduced seed diameter. Consistent with the reduced seed diameter was our observation that the SGP-1

Table 1

Flower swelling power, total starch, and seed traits of EMS-derived lines and controls.

\begin{tabular}{|c|c|c|c|c|c|c|c|}
\hline Population & FSP $N^{\mathrm{c}}$ & FSP $(g / g)^{d}$ & Total starch $N^{\mathrm{c}}$ & Total starch (\%) & Seed weight $N^{c}$ & Seed weight $(\mathrm{mg})^{\mathrm{f}}$ & Seed size $(\mathrm{mm})^{\mathrm{f}}$ \\
\hline SGP-1 wild-type ${ }^{a}$ & 24 & $8.4 \pm 0.10 a$ & 3 & $57.3 \pm 2.47 a$ & 3 & $33.2 \pm 0.51 a$ & $2.90 \pm 0.04 a$ \\
\hline SGP-A1 null ${ }^{\mathrm{a}}$ & 24 & $7.5 \pm 0.10 b$ & 3 & $48.5 \pm 10.58 \mathrm{ab}$ & 3 & $31.7 \pm 0.71 b$ & $2.85 \pm 0.03 a$ \\
\hline EMS M 1 Mountrail/PI 330546 & 294 & $7.3 \pm 0.29 b$ & & & & & \\
\hline DHA175 & 2 & $5.8 \pm 0.15 c$ & 3 & $39.1 \pm 6.50 b$ & 3 & $31.6 \pm 0.76 b$ & $2.73 \pm 0.02 b$ \\
\hline EMS $M_{1}$ Mountrail/IG 86304 & 196 & $7.7 \pm 0.05 b$ & & & & & \\
\hline DHA55 $5^{\mathrm{b}}$ & 2 & $6.4 \pm 0.20 c$ & 3 & $38.5 \pm 3.71 b$ & & & \\
\hline
\end{tabular}

Means \pm standard errors followed with the same letter are not significantly different at $P<0.05$ based on a students $T$-test.

a $\mathrm{F}_{5}$ derived lines from Mountrail/PI 330546.

b SGP-1 double nulls.

c $N=$ number of lines used in analysis.

d FSP = flour swelling power measured on whole grain flour in water/flour suspension ( $\mathrm{g}$ ) over weight of flour ( $\mathrm{g}$ ).

e Total starch was determined on whole grain flour.

f Seed weight and size was determined using the single kernel characterization system on three reps of 200 seeds each. 


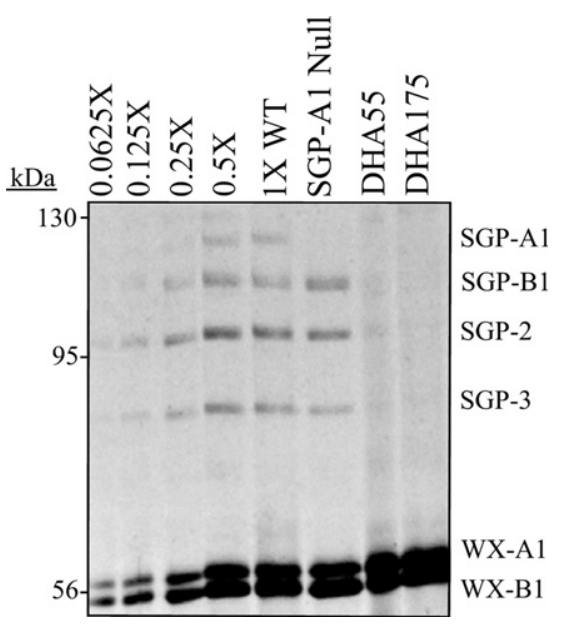

Fig. 1. SDS-PAGE analysis of starch granule proteins from SGP-1 wild-type (WT) and SGP-A1 null (A null) $F_{5}$ derived lines from Mountrail/PI 330546, and SGP-1 double null lines DHA175 and DHA55. The acrylamide gel was silver stained and a dilution series of WT was used to create the loading curve. In lines DHA175 and DHA55 the elimination of both SGP-1 proteins results in reduced binding of SGP-2 and SGP-3.

double null seeds were of similar length to controls but were poorly filled and wrinkled in appearance. Total starch was reduced in both the SGP-A1 nulls and the SGP-1 double nulls with the greatest reductions seen in the SGP-1 double nulls.

\subsection{PCR screening for mutations in SSIIa-A and SSIIa-B}

In the parental SGP-A1 null lines PI 330546 and IG 86304, a 29 bp deletion in SSIIa-A was discovered in the first exon at position 145-174 using the primer set Sgp-A1F3/Sgp-A1R3 (Shimbata et al., 2005). In line DHA175 a point mutation in SSIIa-B was found in the third exon at position 979 where a $G$ to $A$ transition occurred using the primer set Sgp-B1F1/Sgp-B1R1 (Sestili et al., 2010a). This changed the 327th amino acid from aspartic acid (GAT) to asparagine (AAT). In line DHA55, a point mutation was found in SSIIa-B in the eighth exon at position 1864 using the primer set Sgp-B1F2/ Sgp-B1R2 (Shimbata et al., 2005). This was also a G to A transition that resulted in an aspartic acid (GAC) to asparagine (AAC) change in amino acid 622.

\subsection{Microscopic analysis}

Starch from the SGP-1 wild-type line had A-type granules that were smooth and lenticular shaped and B-type granules that were spherical and smooth (Fig. 2). In the SGP-A1 null, the A-type starch granules had a range of minor deformities with the B-type granules similar to those of the wild-type (Fig. 2). In the SGP-1 double null lines, DHA175 and DHA55, both the A and B type granules were deformed and less plump than in the wild-type and SGP-A1 null samples, and had rough or cracked surfaces (Fig. 2).

\subsection{Differential scanning calorimetry analysis and colorimetric amylose content}

The gelatinization properties and amylose content of SGP-1 double null and control starches were examined using DSC. The combined heat scan thermogram shows a clear alteration in the gelatinization of amylopectin in the SGP-1 double nulls (Fig. 3). The SGP-1 double nulls also had a significantly lower gelatinization temperature based on peak temperature and a smaller enthalpy (Fig. 3, Table 2). These data indicate a disruption in amylopectin synthesis and/or composition. The second peak around $105{ }^{\circ} \mathrm{C}$ which is associated with amylose gelatinization was similar in shape and size across all samples with the SGP-1 double null lines having cooler gelatinization temperatures and larger enthalpies compared to the controls (Fig. 3, Table 2). Amylose content in the SGP-A1 null was unchanged compared to the wild-type control whereas the SGP-1 doubles null lines had significantly higher amylose content as determined by both DSC and a colorimetric assay (Table 2).

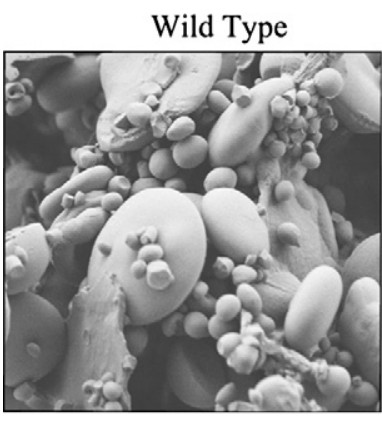

Wild Type

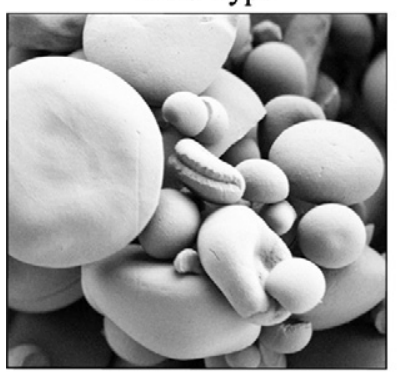

SGP-A1 Null

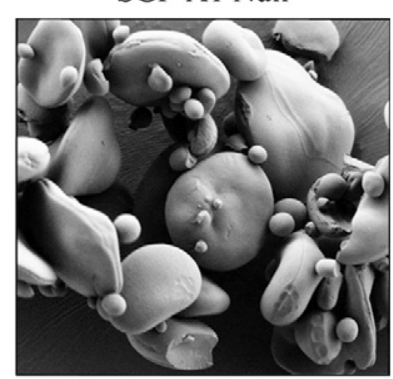

$\underline{20 \mu \mathrm{m}}$

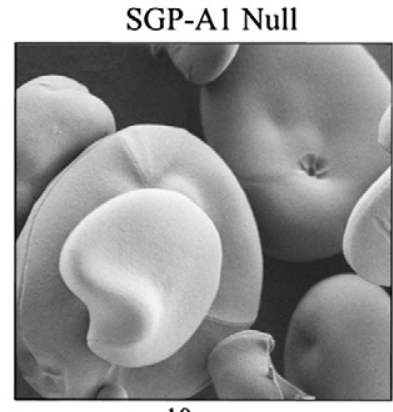

$10 \mu \mathrm{m}$
DHA175

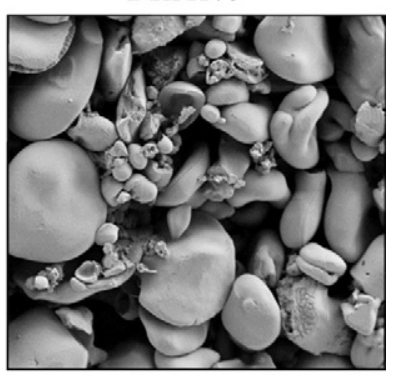

DHA175

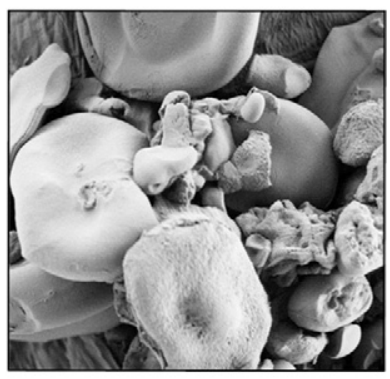

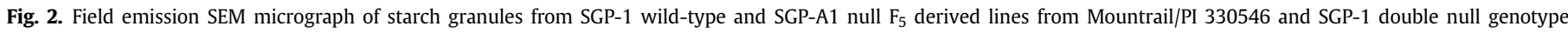
DHA175. 


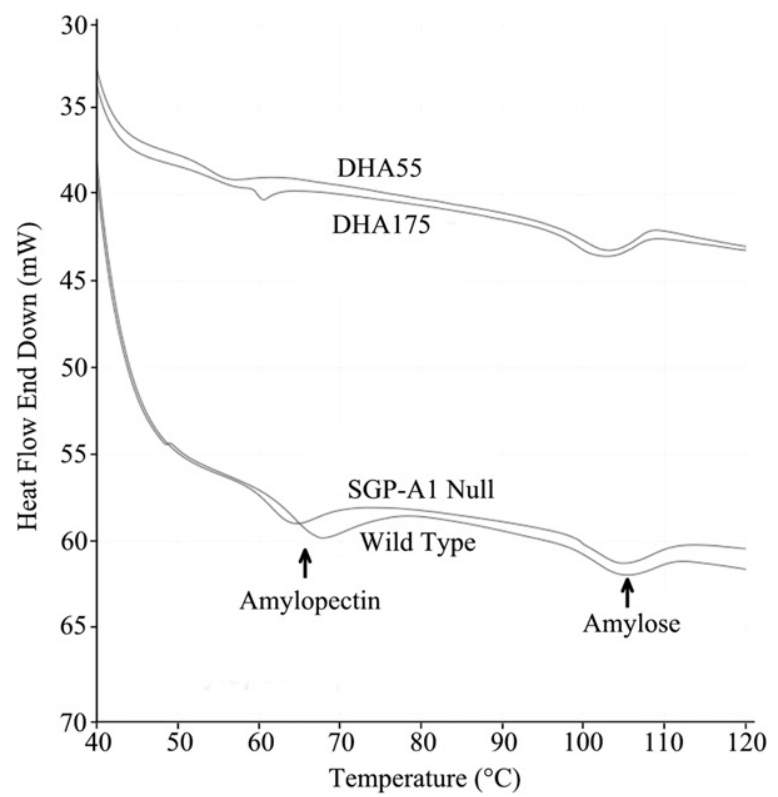

Fig. 3. Differential scanning calorimetry thermogram of starches from SGP-1 wild-type and SGP-A1 null $F_{5}$ derived lines from Mountrail/PI 330546, and SGP-1 double null lines DHA175 and DHA55. SGP-1 double null lines show an altered amylopectin gelatinization profile that occurs at cooler temperatures and has decreased enthalpy compared to the wild-type and SGP-A1 null controls.

\subsection{Starch biosynthetic gene expression analysis}

RNA-Seq data from the two SGP-1 double nulls was combined and compared to Mountrail (SGP-1 wild-type) (Table 3). The SSIIa-A deletion in both SGP-1 double nulls was associated with a dramatic reduction in SSIIa- $A$ transcripts (Table 3 ). Due to the high homology of the SSIIa- $A$ and SsIIa- $B$ genes, the few number of hits detected for SSIIa- $A$ may have arisen from areas where the two genes are $100 \%$ identical. The two independent point mutations in SSIIa-B did not produce the same effect as the deletion in SSIIa-A. On the contrary, there was a significant up regulation of SSIIa-B (Table 3 ). Significant up regulation of transcripts in the SGP-1 double nulls was also exhibited for $W x-A 1$, SsI-1, SbeI-A, SbeIIa-A, SbeIIa-B, SSIII, the large subunit of AGPase, and Pho1.

\section{Discussion and conclusions}

The goal of this investigation was to develop a high-amylose durum line through the mutagenesis of SSIIa (SGP-1). There is little natural variation at this locus as it is a key starch biosynthetic enzyme and screening of $255 \mathrm{~T}$. durum accessions identified only two SGP-A1 nulls and no SGP-B1 nulls. Interestingly, the two lines that were SGP-A1 null, PI 330546 and IG 86304, carried the same
29 bp deletion located in the first exon. This deletion seemingly produces an unstable mRNA as there was a significant reduction of its transcript levels in the two SGP-1 double null lines. This is not the same deletion that was reported by Shimbata et al. (2005) for the SGP-A1 mutant in bread wheat (Yamamori and Endo, 1996). The two separate point mutations created through EMS mutagenesis in $S S I I a-B$ did not produce the same effect; in fact the expression of $S S I I a-B$ was significantly higher in the SGP-1 double null lines compared to the cultivar Mountrail. Neither of the point mutations in SSIIa-B introduced a stop codon but the amino acid changes in DHA175 (D327N) and in DHA55 (D622N) were deleterious to the protein as confirmed by SDS PAGE and the protein prediction software SIFT (Ng and Henikoff, 2003) which gave values of 0.00 and 0.01 respectively $(<0.05$ are considered deleterious). Each of the point mutations also occurred in one of eight highly conserved regions of starch synthases (Li et al., 1999), DHA175 in region 1 and DHA55 in region 5 , indicating they would likely negatively impact enzymatic activity.

As shown in previous studies, several pleiotropic effects were observed as a result of the loss of SSII or SGP-1. As anticipated, the SGP-1 double null lines had significant increases in their amylose content from $38 \%$ to $50 \%(+31 \%)$ using DSC and $28-43 \%(+54 \%)$ using a colorimetric assay. The discrepancy between amylose values obtained from the different methods is a common problem when measuring amylose, and, in general, DSC tends to overestimate amylose content relative to other methods (Zhu et al., 2008). The colorimetric values and percent change in amylose are similar to what was observed previously in durum (Lafiandra et al., 2010) but are higher than those observed in hexaploid wheat (Yamamori et al., 2000; Konik-Rose et al., 2007). Seeds from the SGP-1 double null mutants had a "wrinkled" appearance and, for line DHA175, had a reduced seed size and weight compared to SGP-1 wild-types (Table 1). This is consistent with the literature but a more appropriate comparison would be to create near isogenic lines (NIL) for these mutations as it is possible that the EMS derived lines carry other mutations that could impact seed size. In contrast to our findings, it has been shown in a NIL population that single dose SGP-1 mutations do not impact seed size (Konik-Rose et al., 2007). Appropriately, the SPG-1 double nulls also had a reduced overall starch content compared to SGP-1 wildtypes lines (Table 1 ) as has been reported previously in hexaploid wheat (Yamamori et al., 2000; Konik-Rose et al., 2007) and barley (Morell et al., 2003). However, the reduction in total starch was much more drastic ( $\sim 45 \%$ reduction) than that seen in a hexaploid wheat ( $\sim 20 \%$ reduction). Again, this could partially be attributed to the fact that data presented here are between EMS mutants and non-EMS treated lines as well as different methods were used to measure total starch.

The two SGP-1 double null lines had altered amylopectin gelatinization peaks from the SGP-A1 null and wild-type characterized by a decreased enthalpy and reduced gelatinization

Table 2

Percent amylose and differential scanning calorimetry analysis of SGP-1 double null starches and controls.

\begin{tabular}{|c|c|c|c|c|c|c|}
\hline ID & Amylose (\%) ${ }^{\mathrm{a}}$ & Amylose $(\%)^{b}$ & Peak $1\left({ }^{\circ} \mathrm{C}\right)^{\mathrm{a}}$ & $\Delta \mathrm{H} 1(\mathrm{~J} / \mathrm{g})^{\mathrm{a}}$ & Peak $2\left({ }^{\circ} \mathrm{C}\right)^{\mathrm{a}}$ & $\Delta \mathrm{H} 2(\mathrm{~J} / \mathrm{g})^{\mathrm{a}}$ \\
\hline Wild-type ${ }^{c}$ & $38.0 \pm 0.6 a$ & $28.7 \pm 3.2 \mathrm{a}$ & $64.4 \pm 0.52 a$ & $8.6 \pm 0.72 a$ & $103.8 \pm 0.15 a$ & $4.7 \pm 0.42 b$ \\
\hline SGP-A1 null ${ }^{\mathrm{C}}$ & $39.2 \pm 2.0 \mathrm{a}$ & $31.5 \pm 3.4 a$ & $62.4 \pm 0.44 b$ & $7.8 \pm 0.64 a$ & $102.6 \pm 0.30 b$ & $5.0 \pm 1.06 \mathrm{ab}$ \\
\hline DHA175 & $53.6 \pm 1.1 b$ & $44.3 \pm 5.0 b$ & $57.2 \pm 0.34 c$ & $2.8 \pm 0.46 b$ & $102.8 \pm 0.35 \mathrm{ab}$ & $7.2 \pm 0.25 a$ \\
\hline DHA55 & $48.9 \pm 3.2 b$ & $42.8 \pm 5.2 b$ & $56.2 \pm 0.15 c$ & $2.5 \pm 0.80 b$ & $102.0 \pm 0.40 \mathrm{~b}$ & $6.7 \pm 0.95 \mathrm{ab}$ \\
\hline$P$ value ${ }^{\mathrm{d}}$ & 0.0014 & 0.0043 & $<0.0001$ & 0.0002 & 0.0222 & 0.1180 \\
\hline LSD (0.05) & 6.5 & 8.1 & 1.3 & 2.2 & 1.0 & 2.5 \\
\hline
\end{tabular}

Means \pm standard errors followed with the same letter are not significantly different at $P<0.05$ based on a students $T$-test.

${ }^{a}$ Parameters were determined from thermograms using Pyris 7 DSC software. Values are the mean \pm standard errors ( ) of three biological replicates.

b Determined using a iodine colorimetric assay.

c SGP-1 wild-type and SGP-A1 null $\mathrm{F}_{5}$ derived lines came from Mountrail/PI 330546.

d ANOVA P-value. 
Table 3

RNA-seq expression analysis of starch synthesis genes in developing seeds from SGP-1 null double lines and Mountrail.

\begin{tabular}{|c|c|c|c|c|c|c|}
\hline $\begin{array}{l}\text { Genbank } \\
\text { accession }\end{array}$ & Gene & Mountrail $^{\mathrm{c}}$ & DHA55 ${ }^{c}$ & DHA $175^{c}$ & SGP-1 null ${ }^{\mathrm{d}}$ & SGP-1 Null/WT \\
\hline AJ269503 & Starch synthase II (Ss2a-A) & $876 \pm 57$ & $75 \pm 22$ & $44 \pm 12$ & $59 \pm 19$ & $0.07^{* * *}$ \\
\hline AJ269504 & Starch synthase II (Ss2a-B) & $1145 \pm 117$ & $2477 \pm 370$ & $2020 \pm 180$ & $2249 \pm 297$ & $1.96^{* *}$ \\
\hline AB019622 & Granule-bound starch synthase $I(W x-A 1)$ & $4410 \pm 515$ & $5811 \pm 341$ & $5723 \pm 348$ & $5767 \pm 309$ & $1.31^{*}$ \\
\hline AB019623 & Granule-bound starch synthase $I(W x-B 1)$ & $7180 \pm 811$ & $8046 \pm 740$ & $13,039 \pm 763$ & $10,542 \pm 1716$ & 1.47 \\
\hline AJ292521 & Starch synthase I (SsI-1) & $827 \pm 82$ & $561 \pm 112$ & $936 \pm 145$ & $749 \pm 166$ & 0.91 \\
\hline AJ292522 & Starch synthase I (SsI-2) & $3158 \pm 141$ & $4377 \pm 274$ & $5110 \pm 311$ & $4744 \pm 350$ & $1.50^{* *}$ \\
\hline AF286318 & Starch branching enzyme I-A (Sbe1-A) & $7329 \pm 384$ & $11,694 \pm 1137$ & $14,523 \pm 962$ & $13,109 \pm 1299$ & $1.79^{* *}$ \\
\hline HE591389 & Starch branching enzyme IIa (Sbe2a-A) & $3629 \pm 190$ & $4699 \pm 472$ & $5755 \pm 724$ & $5227 \pm 641$ & $1.44^{*}$ \\
\hline AY740401 & Starch branching enzyme IIa-B (Sbe2a-B) & $1690 \pm 104$ & $2442 \pm 71$ & $2345 \pm 295$ & $2393 \pm 195$ & $1.42^{*}$ \\
\hline AF258608 & Starch synthase III (Ss3) & $700 \pm 27$ & $894 \pm 69$ & $1036 \pm 84$ & $965 \pm 82$ & $1.38^{*}$ \\
\hline AY044844 & Starch synthase IV (Ss4) & $21 \pm 7$ & $37 \pm 7$ & $47 \pm 14$ & $42 \pm 10$ & 1.99 \\
\hline DQ839506 & $\begin{array}{l}\text { ADP-glucose pyrophosphorylase large } \\
\text { subunit (AgpL) }\end{array}$ & $3083 \pm 258$ & $6237 \pm 315$ & $6819 \pm 503$ & $6528 \pm 418$ & $2.12^{* * *}$ \\
\hline AF244997 & $\begin{array}{l}\text { ADP glucose pyrophosphorylase small } \\
\text { subunit (AgpS) }\end{array}$ & $26,631 \pm 3322$ & $20,690 \pm 4399$ & $29,136 \pm 1234$ & $24,913 \pm 3935$ & 0.94 \\
\hline AJ301647 & Isoamylase I (Iso1) & $1730 \pm 74$ & $2211 \pm 232$ & $2113 \pm 285$ & $2162 \pm 235$ & 1.25 \\
\hline EF137375 & Limit dextrinase debranching enzyme I (Ld1) & $1469 \pm 85$ & $1416 \pm 180$ & $2520 \pm 337$ & $1968 \pm 424$ & 1.34 \\
\hline EU595762 & Alpha-1,4-glucan phosphorylase (Pho1) & $1654 \pm 88$ & $2028 \pm 53$ & $2449 \pm 263$ & $2239 \pm 216$ & $1.35^{*}$ \\
\hline U66376 & 1,4-alpha-D-glucanotransferase & $732 \pm 50$ & $874 \pm 144$ & $1311 \pm 157$ & $1093 \pm 193$ & 1.49 \\
\hline JF736013 & HMW glutenin subunit (Glu-B1 Bx7) & $30,040 \pm 3463$ & $27,288 \pm 6732$ & $45,134 \pm 2445$ & $36,211 \pm 7236$ & 1.21 \\
\hline HQ61989a & LMW glutenin subunit (LMW-5) & $675,506 \pm 98,596$ & $461,181 \pm 29,247$ & $1,300,483 \pm 86,856$ & $880,832 \pm 271,666$ & 1.30 \\
\hline AF262983 & Cyclophilin A-2 (Сyp2) & $2569 \pm 234$ & $3183 \pm 368$ & $2696 \pm 399$ & $2939 \pm 376$ & 1.14 \\
\hline AF262984 & Cyclophilin A-3 (Cyp3) & $954 \pm 84$ & $1311 \pm 169$ & $2102 \pm 241$ & $1706 \pm 312$ & $1.79^{*}$ \\
\hline BK001238 & Ribosomal protein L3A-1 (Rpl3a-1) & $2539 \pm 347$ & $1944 \pm 297$ & $2450 \pm 182$ & $2197 \pm 272$ & 0.87 \\
\hline DQ489316 & GTP-binding protein $(G b p-1)$ & $573 \pm 56$ & $702 \pm 64$ & $791 \pm 79$ & $747 \pm 70$ & 1.30 \\
\hline FN429985 & $\begin{array}{l}\text { Glyceraldehyde-3-phosphate dehydrogenase } \\
\text { (Ga3pd) }\end{array}$ & 25,582 & 25,582 & 25,582 & 25,582 & - \\
\hline$J F 727656^{a}$ & $\begin{array}{l}\text { Ubiquitin-protein ligase/zinc ion binding } \\
\text { protein (Zfp-1) }\end{array}$ & $340 \pm 69$ & $298 \pm 31$ & $450 \pm 61$ & $374 \pm 65$ & 1.10 \\
\hline U76896 & Beta-tubulin 5 (Tubb5) & $1387 \pm 86$ & $1727 \pm 102$ & $1663 \pm 219$ & $1695 \pm 154$ & 1.22 \\
\hline
\end{tabular}

a Tissue of origin was unavailable; all other sequences came from developing endosperms.

b Sequences are from genomic DNA with all introns removed; all other sequences were mRNA derived.

c Mean linear counts \pm standard errors from three biological replicates after normalization to Ga3pd.

d Data from DHA55 and DHA175 was combined $(n=6)$.

e Value is the ratio of SGP-1 null counts over Mountrail (WT) counts. Significance was determined from a two-tailed students $t$-test comparing counts from the combined SGP-1 nulls $(n=6)$ and Mountrail $(n=3),{ }^{*}<0.05,{ }^{* *}<0.01$ and ${ }^{* * *}<0.001$.

temperature (Fig. 3, Table 2). The decreases in enthalpy and gelatinization temperatures likely result from the higher amylose/ amylopectin ratio. Amylopectin provides ordered structure and crystallinity while amylose exists in more amorphous form. The higher ordered structures conveyed by amylopectin require more energy to disrupt (Vladimir et al., 2004). In line DHA55, the peak for amylopectin gelatinization was almost too small to distinguish. Accordingly, the SGP-1 double null lines also had a lower FSP (Table 1). These results concur with previous findings in bread wheat (Yamamori et al., 2006; Konik-Rose et al., 2007) and barley (Morell et al., 2003) and are evidence of a disruption in amylopectin synthesis, and an increase in starch amylose content. Both A and B starch granules from SGP-1 double nulls were deformed and as previously described had rough or cracked surfaces (Yamamori et al., 2000; Morell et al., 2003; Kosar-Hashemi et al., 2007). In bread wheat, SGP-1 null lines showed a reduction in B-type starch granules (Konik-Rose et al., 2007), and while not statistically determined, we also observed an overall decrease in the amount of B-type starch granules in the durum SGP-1 double null lines. As seen in bread wheat (Yamamori et al., 2000), durum wheat (Lafiandra et al., 2010) and barley (Morell et al., 2003), there was an almost complete loss of other starch biosynthetic enzymes from the interior of starch granules, namely SBEII (SGP-2) and SSI (SGP-3), while GBSSI remained intact. The loss of these proteins however, did not mean that these proteins were not produced. It has been shown that in the soluble fraction of the endosperm, SBEII, SSI, and GBSSI accumulate at normal levels (Kosar-Hashemi et al., 2007; Morell et al., 2003). It has been hypothesized that SSs, SBEs, along with other starch biosynthetic enzymes act together in complexes in the wheat amyloplast and when one of these enzymes is disrupted, it has significant effects on the other enzymes (Tetlow et al., 2004) or on their final distribution. In the SGP-1 double null lines, this is manifested by the lack of entrapment of SSI and SBEII in the starch granule matrix. Tetlow et al. (2008) demonstrated that in bread wheat, SBEII, SSI, and SSIla interact to form a complex during starch deposition which is controlled by phosphorylation. The loss of SSII likely restricts the formation of this complex and in turn long-chain amylopectin formation and the entrapment of SBEII and SSI.

Transcript levels of starch biosynthetic genes as measured by RNA-Seq were not reduced in the SGP-1 double nulls and in some cases were up-regulated. $W x-A 1$, SsI-1, SbeI-A, SbelIa-A, SbeIIa-B, SSIII, AgpL (large subunit of AGPase), and Pho1 (alpha-1,4-glucan phosphorylase) transcript levels were increased in the SGP-1 double null lines. The up-regulation of starch biosynthetic genes after the elimination of a key enzyme has also been observed in bread wheat where SbeIIa was silenced using RNAi (Sestili et al., 2010b). Using qRT-PCT, Sestili et al. (2010b) observed increases in $W x-1$, SSIII, Iso1, and Ld1 transcripts but no increase for SsI, SSIIa, SbeIIb, or SbeI. The increase in starch biosynthetic gene related transcripts in the durum SGP-1 double null lines was less than previously observed (Sestili et al., 2010b). However, RNA-seq provides a more precise assessment of transcript numbers than does quantitative RT-PCR. The phenomenon of starch biosynthetic genes being up-regulated when one of the critical genes is absent may result from increased levels of sugars in the absence of full starch biosynthesis. As noted above, NILs for these mutations would be more ideal for studying the effects the SGP-1 null phenotype has on gene expression whereas the data presented here are between EMS-treated lines and a non-mutagenized modern cultivar. There 
could be additional deleterious mutations that effect starch synthesis gene expression residing in these lines.

Given the high amylose content, altered gelatinization properties, and decreased FSP of the SGP-1 double nulls presented here, it is reasonable to assume that there will be significant impact on their end use quality. In fact, when noodles were made from the Mountrail/PI $33038 \mathrm{~F}_{5}$ and Mountrail/IG-88905 $\mathrm{F}_{5}$ populations, an increase in noodle firmness was associated with the SGP-A1 null trait (Martin et al., submitted for publication). The SPG-1 double null lines should produce a more profound effect upon noodle firmness as the SGP-A1 null lines do not have increased amylose content. Along with increased noodle firmness, there is a possibility that these lines will also have potential health benefits. In both human and animal trials, high amylose bread wheat and barley with increased resistant starch was shown to increase overall colon health (Bird et al., 2008; Regina et al., 2006) and produce a lower glycemic index (Halstrom et al., 2011; King et al., 2008).

\section{Acknowledgments}

This research was funded by Dow AgroSciences and the Montana Agricultural Experiment Station.

\section{References}

Bird, A.R., Vuaran, M.S., King, R.A., Noakes, M., Keogh, J., Morell, M.K., Topping, D.L., 2008. Wholegrain foods made from a novel high-amylose barley variety (Himalaya 292) improve indices of bowel health in human subjects. British Journal of Nutrition 99, 1032-1040.

Elias, E.M., Miller, J.D., 2000. Registration of 'Mountrail' durum wheat. Crop Science $40,1499-1500$.

Feiz, L., Martin, J.M., Giroux, M.J., 2009. Creation and functional analysis of new Puroindoline alleles in Triticum aestivum. Theoretical and Applied Genetics 118 247-257.

Hallstrom, E., Sestili, F., Lafiandra, D., Bjorck, I., Ostman, E., 2011. A novel wheat variety with elevated content of amylose increases resistant starch formation and may beneficially influence glycaemia in healthy subjects. Food and Nutrition Research 55, 7074

Hansen, L.E., Jackson, D.S., Wehling, R.L., Wilson, J.D., Graybosch, R.A., 2010. Functionality of native tetraploid wheat starches: effects of waxy loci alleles and amylose concentrations in blends. Journal of Cereal Science 52, 39-45.

Hazard, B., Zhang, X., Colasuonno, P., Uauy, C., Beckles, D.M., Dubcovsky, J., 2012. Induced mutations in the starch branching enzyme II (SBEII) genes increase amylose and resistant starch content in durum wheat. Crop Science 52, 1754-1766.

King, R.A., Noakes, M., Bird, A.R., Morell, M.K., Topping, D.L., 2008. An extruded breakfast cereal made from a high amylose barley cultivar has a low glycemic index and lower plasma insulin response than one made from a standard barley. Journal of Cereal Science 48, 526-530.

Konik-Rose, C., Thistleton, J., Chanvrier, H., Tan, I., Halley, P., Gidley, M., KosarHashemi, B., Wang, H., Larroque, O., Ikea, J., McMaugh, S., Regina, A., Rahman, S., Morell, M.K., Li, Z.Y., 2007. Effects of starch synthase Ila gene dosage on grain, protein and starch in endosperm of wheat. Theoretical and Applied Genetics 115, 1053-1065.

Kosar-Hashemi, B. Li, ZY., Larroque, O., Regina, A Yamamori, Y.M., Morell, M.K. Rahman, S., 2007. Multiple effects of the starch synthase II mutation in developing wheat endosperm. Functional Plant Biology 34, 431-438.
Lafiandra, D., Sestili, F., D’Ovidio, R., Janni, M., Botticella, E., Ferrazzano, G., Silvestri, M., Ranieri, R., DeAmbrogio, E., 2010. Approaches for modification of starch composition in durum wheat. Cereal Chemistry 87, 28-34.

Li, Z., Chu, X., Mouille, G., Yan, L., Kosar-Hashemi, B., Hey, S., Napier, J., Shewry, P., Clarke, B., Appels, R., Morell, M.K., Rahman, S., 1999. The localization and expression of the class II starch synthases of wheat. Plant Physiology 120, 1147-1156.

Marioni, J.C., Mason, C.E., Mane, S.M., Stephens, M., Gilad, Y., 2008. RNA-seq: an assessment of technical reproducibility and comparison with gene expression arrays. Genome Research 18, 1509-1517.

Martin, J.M., Sherman, J.D., Lanning, S.P., Talbert, L.E., Giroux, M.J., 2008. Effect of variation in amylose content and puroindoline composition on bread quality in a hard spring wheat population. Cereal Chemistry 85 (2), 266-269.

Martin, J.M., Hogg, A.C., Hofer, P., Manthey, F.A., Giroux, M.J. Impacts of SSII-A Null Allele on Durum Wheat Noodle Quality. Cereal Chemistry. submitted for publication.

Morell, M.K., Kosar-Hashemi, B., Cmiel, M., Samuel, M.S., Chandler, P., Rahman, S., Buleon, A., Batey, I.L., Li, Z.Y., 2003. Barley sex6 mutants lack starch synthase Ila activity and contain a starch with novel properties. Plant Journal 34, 172-184.

Nugent, A.P., 2005. Health properties of resistant starch. Nutrition Bulletin 30, 27-54.

Ng, P.C., Henikoff, S., 2003. SIFT: predicting amino acid changes that affect protein function. Nucleic Acids Research 31, 3812-3814.

Polaske, N.W., Wood, A.L., Campbell, M.R., Nagan, M.C., Pollak, L.M., 2005. Amylose determination of native high-amylose corn starches by differential scanning calorimetry. Starch/Stärke 57, 118-123.

Regina, A., Bird, A., Topping, D., Bowden, S., Freeman, J., Barsby, T., KosarHashemi, B., Li, Z.Y., Rahman, S., Morell, M., 2006. High-amylose wheat generated by RNA interference improves indices of large-bowel health in rats. Proceedings of the National Academy of Sciences of the USA 103, 3546-3551.

Sestili, F., Botticella, E., Bedo, Z., Phillips, A., Lafiandra, D., 2010a. Production of novel allelic variation for genes involved in starch biosynthesis through mutagenesis. Molecular Breeding 25, 145-154.

Sestili, F., Janni, M., Doherty, A., Botticella, E., D’Ovidio, R., Masci, S., Jones, J.D., Lafiandra, D., 2010b. Increasing the amylose content of durum wheat through silencing of the SBEIIa genes. BMC Plant Biology 10, 144.

Shimbata, T., Nakamura, T., Vrinten, P., Saito, M., Yonemaru, J., Seto, Y., Yasuda, H. 2005. Mutations in wheat starch synthase II genes and PCR-based selection of a SGP-1 null line. Theoretical and Applied Genetics 111, 1072-1079.

Smith, A.M., Zeeman, S.C., 2006. Quantification of starch in plant tissues. Nature Protocols 3, 1342-1345.

Tetlow, I.J., Beisel, K.G., Cameron, S., Makhmoudova, A., Liu, F., Bresolin, N.S., Wait, R., Morell, M.K., Emes, M.J., 2008. Analysis of protein complexes in wheat amyloplasts reveals functional interactions among starch biosynthetic enzymes. Plant Physiology 146, 1878-1891.

Tetlow, I.J., 2006. Understanding storage starch biosynthesis in plants: a means to quality improvement. Canadian Journal of Botany 84, 1167-1185.

Tetlow, I.J., Wait, R., Lu, Z.X., Akkasaeng, R., Bowsher, C.G., Esposito, S., KosarHashemi, B., Morell, M.K., Emes, M.J., 2004. Protein phosphorylation in amyloplasts regulates starch branching enzyme activity and protein-protein interactions. Plant Cell 16, 694-708.

Vladimir, P.Y., Krivandin, A.V., Kiseleva, V.I., Wasserman, L.A., Genkina, N.K., Fornal, J., Blaszczak, W., Schiraldi, A., 2004. Structural parameters of amylopectin clusters and semi-crystalline growth rings in wheat starches with different amylose content. Carbohydrate Research 339, 2683-2691.

Yamamori, M., Endo, T.R., 1996. Variation of starch granule proteins and chromosome mapping of their coding genes in common wheat. Theoretical and Applied Genetics 93, 275-281.

Yamamori, M., Fujita, S., Hayakawa, K., Matsuki, J., Yasui, T., 2000. Genetic elimination of a starch granule protein, SGP-1, of wheat generates an altered starch with apparent high amylose. Theoretical and Applied Genetics 101, 21-29.

Yamamori, M., Kato, M., Yui, M. Kawasaki, M., 2006. Resistant starch and starch pasting properties of a starch synthase Ila-deficient wheat with apparent high amylose. Australian Journal of Agricultural Research 57, 531-535.

Zhu, T.M., Jackson, D.S., Wehling, R.L., Geera, B., 2008. Comparison of amylose determination methods and the development of a dual wavelength iodine binding technique. Cereal Chemistry $85,51-58$. 